\title{
Abordaje prelagrimal del seno maxilar: experiencia del Hospital Clínico de la Universidad de Chile
}

\section{Pre-lacrimal approach of maxillary sinus: experience of University of Chile Clinical Hospital}

\author{
Karen García C. ${ }^{1}$, David Fuentealba D. ${ }^{2}$, Luis Barahona A. ${ }^{1}$, Sofía del Lago K. ${ }^{3}$, Andrés Alvo C. ${ }^{1}$, \\ Rodrigo Cataldo de la C. ${ }^{1}$, Alfredo Naser G. ${ }^{1}$, Rodolfo Nazar S. ${ }^{1}$
}

Departamento de Otorrinolaringología, Hospital Clínico de la Universidad de Chile. Santiago, Chile.

${ }^{2}$ Servicio de Otorrinolaringología, Hospital del Salvador. Santiago, Chile.

${ }^{3}$ Departamento de Pediatría y Cirugía Infantil Oriente, Universidad de Chile. Santiago, Chile.

Los autores declaran no tener conflicto de interés.

Recibido el 3 de agosto de 2019. Aceptado el 7 de Mayo de 2020

Correspondencia: Rodolfo Nazar S. Santos Dumontt 999. Departamento Otorrinolaringología, Hospital Clínico Universidad de Chile. Santiago, Chile. Email: rnazars@gmail.com

\section{Resumen}

Introducción: La patología del seno maxilar es frecuente en la práctica del otorrinolaringólogo. El abordaje prelagrimal permite un amplio acceso al seno maxilar, preservando el cornete inferior y ducto nasolagrimal. Objetivo: describir y analizar las características de los pacientes sometidos a abordaje prelagrimal del seno maxilar, en el Servicio de Otorrinolaringología del Hospital Clínico de la Universidad de Chile (HCUCh). Material y Método: Estudio retrospectivo, descriptivo, incluyendo los pacientes con patología otorrinolaringológica sometidos a abordaje prelagrimal del seno maxilar, entre los años 2015 y 2019 en HCUCh. Se analizaron variables epidemiológicas, clínicas, resultados y complicaciones. Resultados: La serie está constituida por 11 pacientes, con edad promedio de 46,8 años; 6 hombres y 5 mujeres. Los diagnósticos más frecuentes que motivaron esta intervención fueron papiloma invertido y pólipo antrocoanal recidivado. Todos los pacientes fueron estudiados con endoscopía nasal e imágenes, siendo de elección la tomografía computada. No se reportaron complicaciones postoperatorias mayores. En el período de seguimiento, ningún paciente ha requerido reintervención del seno maxilar. Conclusión: El abordaje prelagrimal del seno maxilar es una alternativa para abordar esta cavidad, en pacientes adecuadamente seleccionados. En nuestra experiencia, la tasa de complicaciones es baja y no hemos presentado casos de recidiva. Palabras clave: seno maxilar, cirugía endoscópica nasal, prelagrimal.

\begin{abstract}
Introduction: Maxillary sinus disease is frequent in otorhinolaryngology clinical practice. The pre-lacrimal approach allows a wide access to the maxillary sinus, preserving the inferior turbinate and the nasolacrimal duct. Aim: To describe and analyze the characteristics of patients undergoing endoscopic sinus surgery (ESS) with pre-lacrimal approach to the maxillary sinus at University of Chile Clinical Hospital. Material and Method: Retrospective, descriptive study of all patients who underwent ESS for the treatment of any otorhinolaryngological pathology with pre-lacrimal approach, between 2015 and 2019. Variables analyzed include demographics, signs and symptoms and surgical complications. Results: This series includes 11 patients, with a mean age of 46.8 years at the time of surgery; 6 men and 5 women. The main diagnoses included inverted papilloma and recurrent antrochoanal polyp. All the patients were studied with nasal endoscopy and images, with computed tomography being the modality of choice. No major complications were observed at follow-up. Conclusion: The pre-lacrimal approach to the maxillary sinus is an alternative to access this cavity, especially in well-selected patients. In our experience, the complication rate is low. We report no recurrences so far.
\end{abstract}

Keywords: maxillary sinus, endoscopic sinus surgery, pre-lacrimal. 


\section{Introducción}

La patología del seno maxilar es frecuente y diversa en nuestra práctica clínica. Durante la cirugía endoscópica nasosinusal (CENS) es frecuente realizar uncinectomía y antrostomía maxilar, para tratar la enfermedad del seno maxilar. Sin embargo, en ciertos casos, esto no es suficiente para abordar el seno maxilar de forma adecuada, en particular su pared anterior y lateral, para lo cual puede ser necesario utilizar otro abordaje, como Caldwell Luc, punción de la fosa canina o abordaje transeptal. En 2013, el grupo de Zhou propone el abordaje endoscópico prelagrimal del seno maxilar. Esta técnica permite un amplio acceso al seno maxilar, preservando el cornete inferior y el ducto nasolagrimal (Figura 1) ${ }^{1}$. Su uso se ha reportado en diversas patologías, incluyendo pólipos antrocoanales, papiloma invertido, tumores de las fosas pterigopalatina e infratemporal, entre otros ${ }^{2-5}$. El abordaje prelagrimal permite acceder al seno maxilar por anterior al conducto nasolagrimal en forma oblicua, logrando un adecuado control de las paredes anterior y lateral, con ópticas de 0 y 45 grados.

\section{Objetivo}

Describir y analizar las características clínicas, epidemiológicas y quirúrgicas de los pacientes sometidos a cirugía endoscópica del seno maxilar, con abordaje prelagrimal, en el Servicio de Otorrinolaringología del Hospital Clínico de la Universidad de Chile (HCUCh), junto con describir la técnica utilizada.

\section{Material y Método}

Se realizó un estudio retrospectivo, descriptivo, de todos los pacientes que fueron sometidos a cirugía endoscópica de seno maxilar, con abordaje prelagrimal, entre enero de 2015 y marzo de 2019, en el HCUCh. Se realizó re-
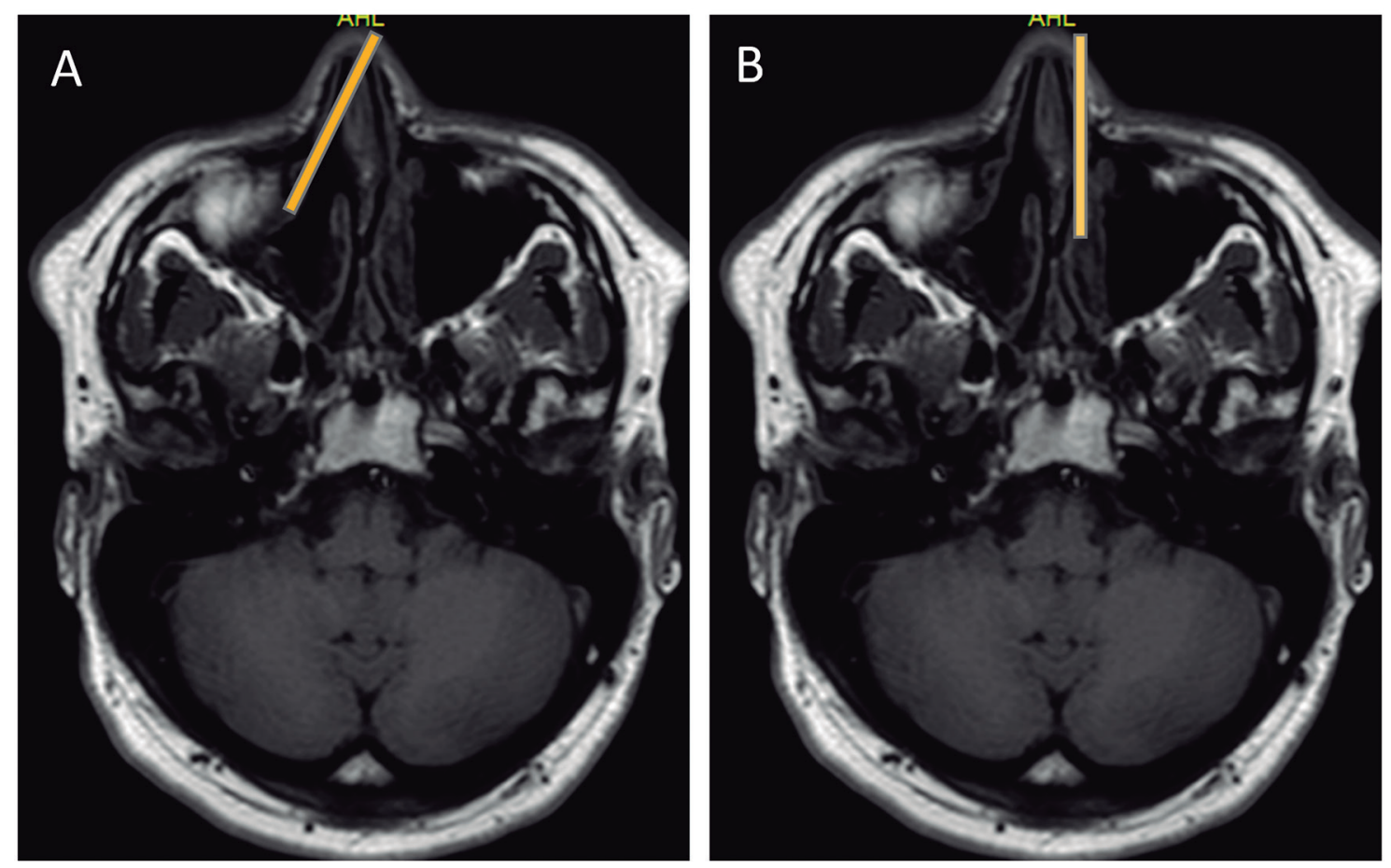

Figura 1. Resonancia magnética postoperatoria que muestra en forma esquemática: (A) En corte axial por fosa nasal derecha, la visión que se logra con el abordaje prelagrimal de todas las paredes del seno maxilar con endoscopio de $45^{\circ}$, con un adecuado control de la pared anterior. (B) En corte axial por fosa nasal izquierda, la visión con endoscopio rígido de $45^{\circ}$, a nivel de antrostomía maxilar tradicional, con limitada visión de la pared anterior. 
visión de ficha clínica electrónica, para rescate y análisis de datos epidemiológicos, clínicos, quirúrgicos y resultados. Todos los pacientes disponen de evaluación preoperatoria con anamnesis, examen físico, endoscopía nasal e imágenes. El análisis estadístico se realizó con Microsoft Excel.

\section{Técnica quirúrgica}

La técnica quirúrgica utilizada se ilustra en la Figura 2.
En todos los pacientes, la cirugía se llevó a cabo con endoscopio de $0^{\circ}$ y $4 \mathrm{~mm}$ de diámetro. Una vez realizado el acceso, se exploró el seno maxilar con ópticas de $0^{\circ}$ y $45^{\circ}$. Los pasos principales de la cirugía son los siguientes:

Antes de comenzar, las fosas nasales se preparan con cotonoides con adrenalina 1:20.000 en el meato medio y en la línea maxilar anterior. La mucosa en la línea maxilar anterior se infiltra con una solución de lidocaína $2 \%$ con adrenalina 1:80.000. Luego se realiza una
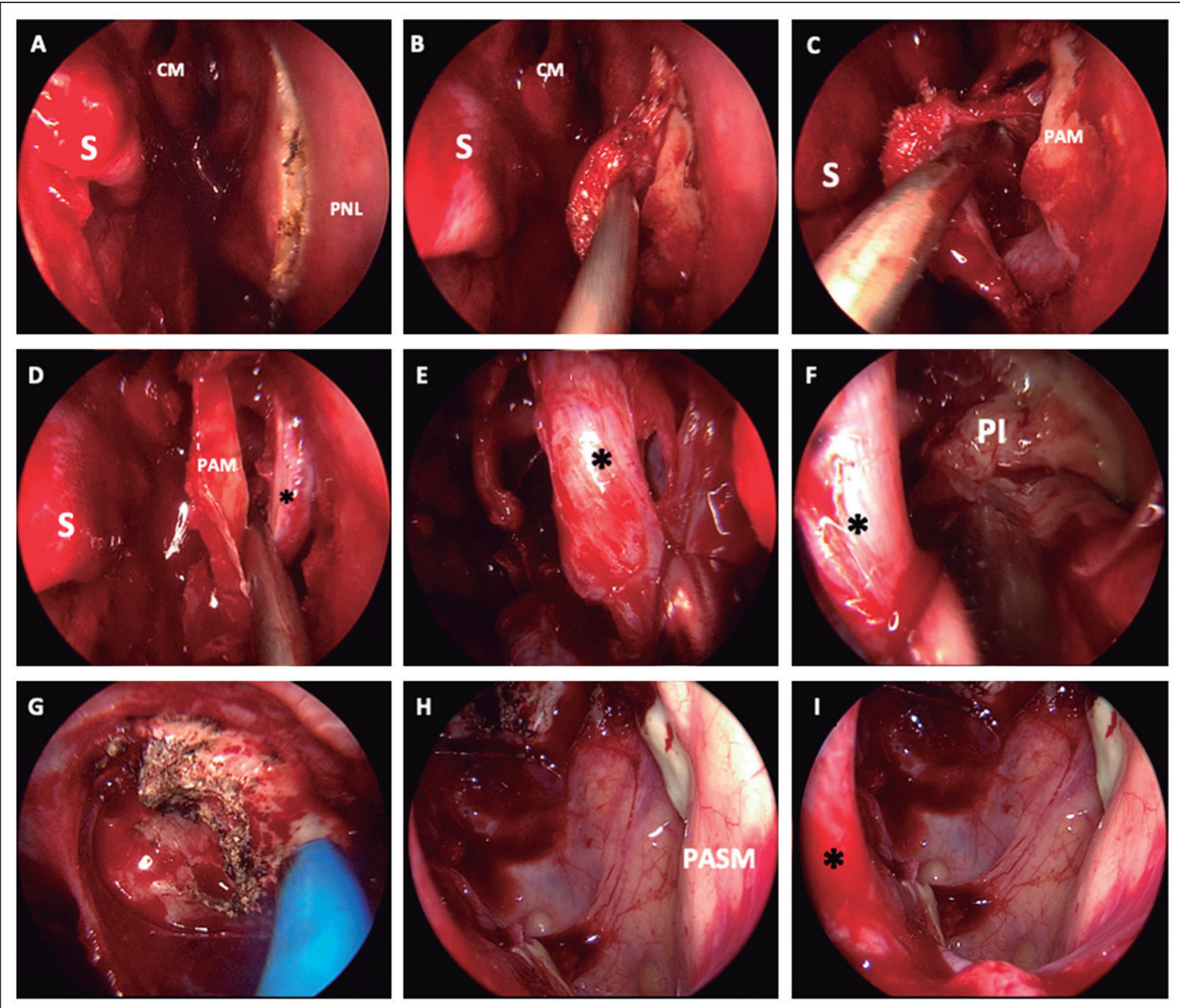

Figura 2. Secuencia de pasos quirúrgicos del abordaje prelagrimal en un paciente con papiloma invertido. Imágenes con endoscopio de 4 mm de $0^{\circ}$ por fosa nasal izquierda. (A) Incisión arciforme en pared nasal lateral. (B) Elevación de colgajo mucoperióstico. (C) Exposición de proceso ascendente del hueso maxilar. (D) Identificación de conducto lagrimal, posterior a osteotomía del proceso ascendente del maxilar. (E) Acceso prelagrimal al seno maxilar con aspirador curvo. (F) Resección con microdebridador de papiloma invertido. (G) Cauterización con aspirador coagulador de la zona de inserción del papiloma en la pared anterolateral del seno maxilar. (H) Control total de la pared anterior del seno maxilar. (I) Visualización del acceso prelagrimal posterior a resección. S: septum; CM: cornete medio; PNL: pared nasal lateral; *conducto nasolagrimal; PAM: proceso ascendente del hueso maxilar; PI: papiloma invertido; PASM: pared anterior del seno maxilar. 
incisión arciforme en la pared lateral de la fosa nasal, entre la escotadura piriforme y la cabeza del cornete inferior, continuándose con la línea lagrimal o maxilar anterior (Figura 2A). Se eleva el colgajo mucoperióstico hacia posterior (Figura 2B), exponiendo la apófisis ascendente del hueso maxilar, sobre el que se realizan osteotomías con cinceles curvos de $3 \mathrm{~mm}$ (Figura 2C). Así, se logra remover parte del proceso frontal del maxilar, creando una ventana ósea hasta lograr una exposición amplia prelagrimal del seno maxilar. Con esto también se expone el conducto nasolagrimal, que se esqueletiza prolijamente para preservarlo (Figuras $2 \mathrm{D}$ y E). Lo anterior permite exponer en forma completa la mucosa del seno maxilar en todas sus paredes. Todo el tejido patológico se remueve bajo visión directa, con pinzas o bien asistido con microdebridador (Figura 2F.) El abordaje permite el acceso a todas las paredes del seno, teniendo especial control de las paredes anterior y lateral. En aquellos pacientes con papiloma invertido o pólipo antrocoanal, se identificó apropiadamente el sitio de inserción y se realizó fresado y cauterización de éste (Figura 2G). Antes de finalizar la cirugía, es posible revisar con endoscopio de $30^{\circ}$ y $45^{\circ}$, con completo control de todas las paredes del seno maxilar (Figuras $2 \mathrm{H}$ y $2 \mathrm{I}$ ). Finalmente, se reposiciona el colgajo mucoso, dejando cubierto el conducto nasolagrimal. Como elección, dejamos espuma hemostática en la fosa nasal, para evitar el taponamiento convencional.

\section{Resultados}

\section{Pacientes}

La serie de casos clínicos está constituida por 11 pacientes: 5 mujeres y 6 hombres. La edad promedio de intervención fue de 46,8 años (rango 18-83 años). En la Tabla 1 se resumen las principales características demográficas, clínicas, quirúrgicas y del seguimiento de los pacientes.

Todos los pacientes cuentan con endoscopía nasal y estudio con imágenes previo a la cirugía. La imagen de elección fue la tomografía computada (TC) de cavidades paranasales (CPN) sin contraste con cortes finos, con cortes coronales, axiales y sagitales.
En tres casos se complementó con resonancia magnética (RM), por sospecha de papiloma invertido, para evaluar la extensión tumoral. En dos casos se solicitó angio-RM por sospecha de lesión vascular y tumor unilateral con extenso compromiso en la TC. La indicación de este abordaje fue en su mayoría por: lesión extensa del seno maxilar, ubicación en pared anterior de la patología y recidiva de papiloma invertido o pólipo antrocoanal.

Las patologías que motivaron la cirugía fueron principalmente el papiloma invertido en cuatro casos y el pólipo antrocoanal recidivado en tres casos, identificándose, además, otros cuatro casos con diagnóstico de neurofibroma, neuroblastoma olfatorio, rinosinusitis crónica y rinosinusitis aguda recurrente. Respecto a estos últimos pacientes es importante mencionar que el caso de neuroblastoma olfatorio se obtuvo como diagnóstico histológico posoperatorio, ya que previo a la cirugía se sospechaba un tumor de estirpe vascular. El neuroblastoma no invadía el seno maxilar, sin embargo, se decide el abordaje prelagrimal, para adecuado control de la arteria maxilar, previo a la resección tumoral. El paciente con rinosinusitis crónica tenía una imagen sugerente de pólipo en la pared anterolateral del seno maxilar, lo cual motivó a realizar este abordaje para acceder adecuadamente a esta zona. Por otra parte, el caso de sinusitis aguda recurrente, tenía una fosa nasal muy estrecha, con anatomía alterada de la pared nasal lateral, por lo que se decide un abordaje anterior prelagrimal, que permitió un adecuado acceso al seno. Cinco pacientes tienen antecedente de una intervención previa en el seno maxilar.

\section{Cirugía}

El tiempo quirúrgico promedio fue de 132 minutos (rango de 65 a 320 minutos). En dos casos, previo al abordaje prelagrimal se realizó uncinectomía y antrostomía maxilar. En un solo caso se realizó una ventana septal para asistir la cirugía, correspondiente al paciente con diagnóstico de neurofibroma. No se reportaron complicaciones durante la cirugía, a excepción del paciente número 9 descrito en la Tabla 1, que presentó un sangrado abundante en el intraoperatorio y requirió la transfusión de 2 unidades de glóbulos rojos, con buena evolución posterior. 


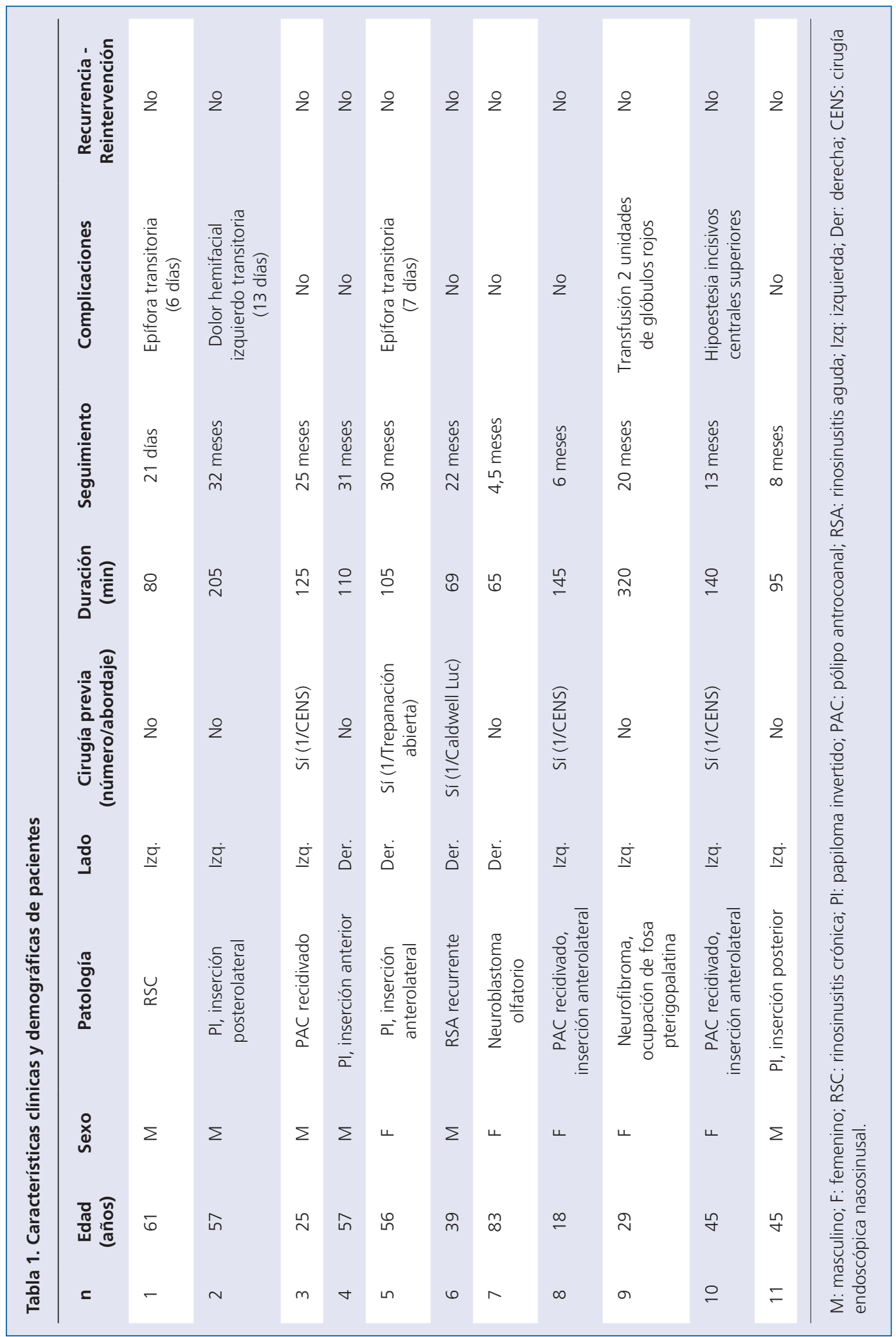




\section{Postoperatorio}

La mayoría de los pacientes fueron dados de alta el día siguiente, excepto del sujeto con diagnóstico de neurofibroma, siendo dado de alta al cuarto día postoperatorio. Todos los pacientes recibieron indicaciones para el postoperatorio, incluyendo el uso de lavados nasales de alto flujo y corticoesteroides tópicos a partir del día 15.

Los hallazgos histológicos de la biopsia diferida fueron compatibles con el diagnóstico preoperatorio en la mayoría de los casos, a excepción del paciente con neuroblastoma olfatorio, que al examen endoscópico y resonancia preoperatoria se sugería un tumor vascular con sospecha de hemangiopericitoma, decidiéndose el abordaje prelagrimal para optimizar la visualización del tumor para acceder a la pared posterior del seno maxilar. Este paciente, posteriormente, realizó radioterapia y se mantuvo libre de enfermedad durante el seguimiento realizado.

El seguimiento se realizó con endoscopía a los días 15 y 30, y luego a los 3, 6 y 12 meses desde la cirugía. Posteriormente, se continúa con controles cada 6-12 meses en pacientes con lesiones tumorales. En la Figura 3 se evidencia el control endoscópico de un paciente posterior a 15 meses de la cirugía.

El tiempo de seguimiento para esta serie tuvo un promedio de 17,47 meses (21 días a 32 meses), presentando mejoría clínica sintomática en todos los pacientes. Dos pacientes presentaron epífora leve en el primer control, que cedió en forma espontánea al segundo control. Otras complicaciones postoperatorias menores incluyeron a un paciente con dolor hemifacial izquierdo y un paciente con hipoestesia de ambos incisivos centrales. Ambos escenarios se pesquisaron solo al primer control y luego cedieron en forma espontánea. Dado que en ningún paciente en esta serie se ha constatado recurrencia, no se ha requerido reintervención en el tiempo de seguimiento.

\section{Discusión}

Existen múltiples escenarios en que la antrostomía maxilar endoscópica tradicional no permite acceder de manera adecuada a todos los márgenes del seno maxilar. Es por esto

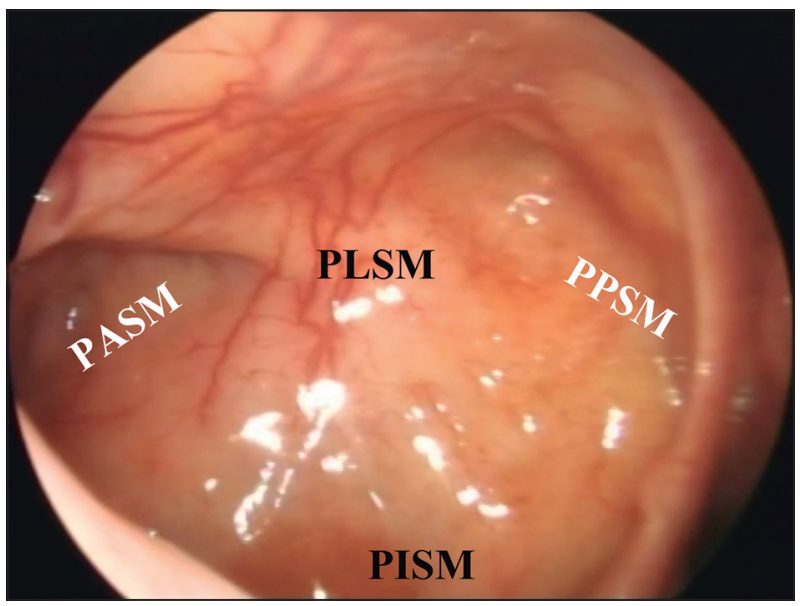

Figura 3. Control endoscópico a los 15 meses postoperatorio del paciente número 4 de la Tabla 1, por fosa nasal derecha. Con óptica de $45^{\circ}$, se evidencia sin dificultad todas las paredes del seno maxilar, sin evidencia de recurrencia. PASM: pared anterior del seno maxilar; PLSM: pared lateral del seno maxilar; PPSM: pared posterior del seno maxilar; PISM: pared inferior del seno maxilar.

que se han planteado múltiples alternativas de abordaje que permiten una visualización más amplia y mejor maniobrabilidad para patologías de esta cavidad, entre ellas el abordaje de Caldwell-Luc, transeptal, la trepanación de la fosa canina, maxilectomía medial endoscópica $y$, recientemente, el abordaje prelagrimal. Se ha visto que hasta un $75 \%$ de los pacientes en que se realiza trepanación de la fosa canina experimentan complicaciones como edema geniano (38\%), dolor facial (32\%), hipoestesia facial $(30 \%)$ o dental $(27 \%)$, dolor geniano $(27 \%)$, entre otras, siendo persistentes hasta el $28 \%$ de los casos $^{6}$.

Recientemente se publicó un artículo en que se compararon dos grupos de pacientes con patología de seno maxilar, que fueron sometidos a un abordaje prelagrimal o a Caldwell-Luc. En ambos grupos se logró la extracción completa de la lesión, sin haber diferencias significativas en el tiempo operatorio, sangrado, estadía hospitalaria ni incidencia de eventos adversos. Estos últimos eventos se limitaron a hipoestesia geniana y labial superior, con una duración menor a 3 meses en los pacientes sometidos a un abordaje prelagrimal, en comparación con el grupo sometido a Caldwell-Luc en que persistió por más de 3 meses en la mayoría de los pacientes, e incluso más de 
un año en 2 casos $^{7}$. Esta diferencia estaría dada por la lesión de ramos del nervio infraorbitario como el alveolar superior anterior y medio en el abordaje prelagrimal, en comparación con la eventual injuria del ramo principal del nervio infraorbitario en el abordaje Caldwell-Luc ${ }^{7}$.

El abordaje prelagrimal, además, tiene la ventaja de preservar el cornete inferior y el conducto nasolagrimal, en comparación con la maxilectomía endoscópica medial clásica que puede llevar a epífora en un 30\% y síndrome de nariz vacía en un $20 \%$ de los casos $^{8}$. En el HCUCh, el abordaje prelagrimal del seno maxilar comenzó a implementarse desde el año 2017, para evitar el uso de incisiones externas en el manejo de diversas patologías que requerían un control de las paredes anterolaterales del seno. En nuestra casuística, la principal indicación corresponde a papiloma invertido, lo cual concuerda con la literatura. En una serie de 15 pacientes con abordaje prelagrimal, recientemente publicada, el principal diagnóstico que motivó esta indicación fue también el papiloma invertido, de los cuales seis casos fueron cirugías de revisión ${ }^{2}$. Otros estudios han demostrado que esta técnica es segura y efectiva tanto para papiloma invertido primario como secundario, particularmente si su pedículo se inserta en la pared antero-inferior, ínfero-lateral del seno maxilar o con inserciones múltiples ${ }^{3,4}$. Otra indicación frecuente en la literatura, así como en nuestra serie, es su uso en pólipos antrocoanales recidivados ${ }^{5}$. $\mathrm{La}$ CENS tradicional tiene una tasa de recurrencia para esta patología, que varía de $0 \%$ a $50 \%{ }^{9,10}$. Los pacientes de esta serie incluidos con este diagnóstico no han presentado recurrencia. Planteamos este abordaje en casos de pólipos antrocoanales recidivados, para asegurar la exposición y manejo del sitio de inserción de forma segura.

En cuanto a la técnica quirúrgica, destacamos la ventaja que otorga al permitir identificar detalladamente el sitio de inserción de la lesión, con la ayuda del endoscopio. Los hallazgos intraoperatorios se deben correlacionar con las imágenes previas, disponibles en pabellón. Algunos autores plantean comenzar la cirugía con uncinectomía y antrostomía maxilar, pese a que en la técnica tradicional no se describe, y luego continuar con el abordaje prelagrimal, argumentando que otorgaría un mejor drenaje de la cavidad, un acceso más amplio para los controles operatorios y mejor penetración de los medicamentos indicados en forma tópica durante el postoperatorio ${ }^{4,5,11}$. En nuestra experiencia, solo en dos casos se realizó de esta manera, mientras que en la gran mayoría se comenzó el abordaje prelagrimal del seno maxilar, para luego continuar de anterior a posterior con la resección de la apófisis unciforme y de la fontanela posterior.

Un estudio reciente realizado en cadáveres evidenció que la adición de una pequeña ventana transeptal al abordaje prelagrimal para permitir el paso de instrumentación angulada de $45^{\circ}$, podría aumentar la accesibilidad al receso alveolar, receso cigomático y a la porción del piso orbitario lateral al nervio infraorbitario ${ }^{12}$. Sin embargo, no existen estudios comparativos que demuestren esta ventaja en pacientes vivos con patología del seno maxilar. En nuestra casuística se realizó una ventana septal solo en un paciente con neurofibroma extenso, siendo uno de los primeros casos sometidos al abordaje prelagrimal en nuestro centro. Con el aumento en el número de pacientes operados con este abordaje, consideramos que en general es innecesaria la confección de una ventana septal por la amplia visualización y maniobrabilidad percibida con el acceso prelagrimal, evitando así la manipulación innecesaria del tabique. En nuestra experiencia, este abordaje permite una amplia exposición de todo el seno maxilar y su patología, sin dejar puntos ciegos, logrando un control completo por parte del otorrinolaringólogo, facilitando la remoción de las lesiones en forma segura, siempre bajo visión directa. Por otro lado, permite el uso del endoscopio de $0^{\circ}$ durante toda la cirugía, lo cual facilita su ejecución. El endoscopio de $45^{\circ}$ se reserva para la revisión de la pared anterolateral del seno.

Si bien la mayor utilidad de este abordaje estaría dada en casos de patología del seno maxilar en sus aspectos anterior e inferior, de difícil acceso para el abordaje endoscópico tradicional, también existe experiencia satisfactoria en lesiones que ocupan el resto de las paredes, la fosa pterigopalatina e infratemporal e incluso algunos autores plantean su uso en patología inflamatoria del seno maxilar como la rinosinusitis crónica. Sin embargo, no existen estudios que comparen el abordaje prelagrimal con otros abordajes tradicionales 
del seno maxilar con esta última indicación ${ }^{13}$.

Las complicaciones secundarias a esta técnica son poco frecuentes y en su mayoría leves. En la mayor serie publicada hasta la fecha con 71 casos, se evidenció que hasta un $7 \%$ podría presentar hipoestesia del labio superior y del ala nasal, y un 5,63\% un colapso nasal leve ${ }^{3}$. Los déficits sensoriales serían transitorios en la mayoría de los casos, cediendo antes de los 6 meses desde la cirugía, como se observa en nuestra casuística y que es consistente con otras series recientes ${ }^{7,13}$. Otras complicaciones incluyen la aparición de vesículas quísticas y granulomas en la cavidad. Al manejarlas con remoción bajo endoscopía y lavados nasales, éstas desaparecen en 2-4 semanas. También se describe la presencia de sinequias ${ }^{4,5}$. No tuvimos desarrollo de sinequias en nuestra casuística, lo cual puede ser atribuido a una reposición adecuada del colgajo y a la irrigación precoz con soluciones salinas. Si bien el conducto nasolagrimal debe preservarse, este puede ser manipulado durante la cirugía, lo cual no se correlaciona siempre con desarrollo de epífora 5 .

En nuestra serie, no se reportaron complicaciones intraquirúrgicas derivadas de este abordaje. En forma diferida, cuatro pacientes presentaron complicaciones menores que cedieron en forma espontánea, mencionadas previamente, por lo que en nuestra serie de casos el abordaje prelagrimal mostró ser una técnica segura y sin morbilidad importante. Sin embargo, se desconoce si este abordaje tiene un impacto en la resistencia del flujo respiratorio y aclaramiento mucociliar a largo plazo, por lo que se requieren estudios de mayor seguimiento a futuro ${ }^{13}$.

En el caso de los papilomas invertidos, existe un riesgo de recurrencia promedio de $10 \%-25 \%$ con las distintas técnicas, que se ha visto que podría reducirse a un $0 \%$ a $7 \%$ con el abordaje prelagrimal ${ }^{3,4,8,14,15}$. El tiempo en que se pesquisa la recurrencia en casos de papiloma invertido varía de meses a años, encontrándose la mayoría en un plazo de 2 años posoperatorio, pero describiéndose casos hasta 8 años. Es por esto que algunos autores recomiendan la necesidad de seguimiento mínimo de 5 años en estos pacientes ${ }^{15}$. El seguimiento se realiza en todas las series con controles endoscópicos seriados en intervalos variables de tiempo.
Algunos autores han realizado el seguimiento únicamente con endoscopía rígida de $0^{\circ}, 45^{\circ}$ y/o $70^{\circ}$, limitando el uso de imágenes como TC o RM en caso de sospecha clínica para evaluar eventuales mucoceles o recurrencia ${ }^{4,8,13}$. Otros autores realizan el seguimiento principalmente con endoscopía flexible ${ }^{14}$, mientras que otros añaden incluso un control tomográfico cada 6 meses en el primer año y anual luego de este periodo ${ }^{3}$. No existen estudios comparativos respecto al seguimiento, por lo que no existe un seguimiento único correcto. Nosotros recomendamos realizar seguimiento endoscópico seriado, realizando imágenes como TC o RM únicamente en casos en que no se pueden visualizar las paredes del seno maxilar en el seguimiento en pacientes con reinicio o deterioro de sintomatología. En la Figura 3 se puede apreciar una imagen obtenida con una óptica angulada en el seguimiento de un paciente operado con abordaje prelagrimal del seno maxilar, en donde es posible identificar todas las paredes de esta cavidad.

Esta revisión cuenta con algunas limitaciones como su carácter retrospectiva con ausencia de un grupo control, por lo que no se pueden establecer comparaciones con otras técnicas quirúrgicas. Por otro lado, el número de pacientes en esta serie es pequeño y con un periodo de seguimiento corto, principalmente explicado por ser una técnica de reciente uso en nuestro centro, lo que podría ser un aspecto relevante, particularmente en los casos de tumores como los papilomas invertidos que requieren un seguimiento a largo plazo. Esta publicación corresponde, hasta la fecha, a la mayor casuística de abordaje prelagrimal para la patología del seno maxilar reportada en literatura hispana y la segunda en ser publicada, luego de una experiencia mexicana de 6 casos en que se realizó un seguimiento promedio de 14,5 meses y en que no se evidenciaron complicaciones ni recurrencias ${ }^{16}$.

\section{Conclusión}

El abordaje prelagrimal del seno maxilar es una técnica de cirugía endoscópica nasosinusal mínimamente invasiva, reproducible, segura y efectiva en el manejo de patología del seno maxilar. Debe indicarse en base a la etiología, 
ubicación y extensión de la patología que involucra el seno maxilar. Es una herramienta con la cual el otorrinolaringólogo debe estar familiarizado para llevar a cabo en los casos que lo ameriten. La casuística presentada mostró una baja tasa de complicaciones menores y sin recurrencias. Esperamos que este procedimiento se siga realizando con mayor frecuencia en nuestro hospital, en la medida que sea necesario. Esperamos también que se difunda su uso en otros centros, debido a las ventajas ya discutidas, con control excelente de todas las paredes del seno maxilar durante la cirugía endoscópica nasal.

\section{Bibliografía}

1. Zhou B, Han DM, Cui SJ, Huang Q, Wang CS. Intranasal endoscopic prelacrimal recess approach to maxillary sinus. Chin Med J (Engl). 2013; 126(7): 1276-80.

2. Lin YT, Lin CF, Yeh TH. Application of the endoscopic prelacrimal recess approach to the maxillary sinus in unilateral maxillary diseases. Int Forum Allergy Rhinol. 2018; 8(4): 530-536.

3. Zhou B, Huang Q, Sun J, Li X, et al. Resection of Inverted Papilloma of the Maxillary Sinus via a Prelacrimal Recess Approach: A Multicenter Retrospective Analysis of Surgical Efficacy. Am J Rhinol Allergy. 2018 Nov; 32(6): 518-525.

4. Yu QQ, Guan G, Zhang NK, et al. Intranasal endoscopic prelacrimal recess approach for maxillary sinus inverted papilloma. Eur Arch Otorhinolaryngol. 2018; 275(9): 2297-2302.

5. Comoglu S, Celik M, Enver N, Sen C, Polat B, Deger K. Transnasal prelacrimal recess approach for recurrent antrachoanal polyp. J Craniofac Surg. 2016; 27: 1025-1027.

6. Robinson SR, Baird R, Le T, Wormald PJ. The incidence of complications after canine fossa puncture performed during endoscopic sinus surgery. Am J Rhinol. 2005; 19(2): 203-206.

7. Lee JJ, Ahmad Z AM, Kim D, et al. Comparison Between Endoscopic Prelacrimal Medial Maxillectomy and Caldwell-Luc Approach for Benign Maxillary Sinus Tumors. Clin Exp Otorhinolaryngol. 2019;12(3):287-293.

8. Suzuki M, Nakamura Y, Yokota M, Ozaki S, Murakami S. Modified transnasal endoscopic medial maxillectomy through prelacrimal duct approach. Laryngoscope. 2017; 127(10): 2205-2209.

9. Nikakhlagh S, Rahim F, Saki N, Mohammadi H, Maliheh YM. Antrochoanal polyps: report of 94 cases and review the literature. Niger J Med. 2012; 21(2):156-159.

10. Choudhury N, Hariri A, Saleh H. Endoscopic management of antrochoanal polyps: a single UK centre's experience. Eur Arch Otorhinolaryngol. 2015; 272(9): 2305-2311.

11. Morrissey DK, Wormald PJ, Psaltis AJ. Prelacrimal approach to the maxillary sinus. Int Forum Allergy Rhinol. 2016; 6: 214-218.

12. Khong GC, Medikeri G, Tierney C, Leong SC. Adjunctive techniques to improve access of the endoscopic prelacrimal recess approach. Laryngoscope. 2019; 10.1002/lary.28259.

13. Lin YH, Chen WC. Clinical outcome of endonasal endoscopic prelacrimal approach in managing different maxillary pathologies. PeerJ. 2020;8:e8331.

14. Hildenbrand T, Weber R, Mertens J, Stuck BA, Hoch $\mathrm{S}$, Giotakis E. Surgery of Inverted Papilloma of the Maxillary Sinus via Translacrimal Approach-LongTerm Outcome and Literature Review. J Clin Med. 2019; 8(11): 1873.

15. Nygren A, Kiss K, von Buchwald C, Bilde A. Rate of recurrence and malignant transformation in 88 cases with inverted papilloma between 1998-2008. Acta Otolaryngol. 2016; 136(3): 333-336.

16. Sánchez JR, Alanís AJ, Romo JA. Abordaje endoscópico endonasal del receso prelagrimal: serie de casos. An Med (Mex). 2019; 64(4), 260-264. 